\title{
Vom deutsch-französischen Krieg zum Zweiten Weltkrieg
}

\author{
Kriegsberichterstattung zwischen journalistischen Routinen und politischer \\ Propaganda
}

\section{Rudolf Stöber}

Wie groß ist in der Kriegsberichterstattung der Grad journalistischer Autonomie? Quellen, die diese Frage explizit klären würden, sind rar. Mit der Methode der historischen Quellenkritik überprüft der Aufsatz die Frage für die letzten drei großen Kriege, die Deutschland fübrte (1870/71, 1914-18 und 1939-45). Input und Verarbeitung von Presseorganen mit unterschiedlichen Parteiprogrammen werden verglichen. Ein Indizienbeweis untersucht anhand dreier zentraler Medienfunktionen (Informations-, Meinungsbildungs-und Orientierungsfunktion) den journalistischen Gestaltungsspielraum. Dabei zeigen sich signifikante Unterschiede, die sich durch die maßgebliche Randbedingung der Kriegsberichterstattung, das tangierte politische Interesse, erklären lassen. Generell gilt: Nicht Zensur schränkt Kriegsberichterstattungsautonomie am weitesten ein, sondern positive Pressepolitik - v. a. wenn sie journalistische Routinen nutzt. Im Ausblick werden Konsequenzen der Kriegsberichterstattung für das journalistische System erörtert.

Schlagwörter: Kriegsberichterstattung, Pressepolitik, Medienfunktionen, Journalismus, Propaganda, Mediengeschichte

\section{Vorbemerkungen}

\section{A. Fragestellung, Untersuchungsbereich, Methode}

Seit Menschen Krieg führen, ist Kriegsberichterstattung seine unvermeidliche Begleiterscheinung. In den letzten, nicht eben friedlichen Jahren hatte dementsprechend auch die kommunikationswissenschaftliche Behandlung des Themas Konjunktur. ${ }^{1}$ Häufig standen Überlegungen im Vordergrund, die auf die Diskrepanz zwischen der Wirklichkeit des Krieges und ihrem Abbild in der Berichterstattung abhoben. Dabei wird Kriegsberichterstattung zumeist als operative Funktion des Journalismus angesehen; Journalismus im Allgemeinen und Kriegsberichterstattung im Besonderen seien nur systemtheoretisch-konstruktivistisch zu interpretieren: „Die mediale Wirklichkeit des Krieges ist demnach nicht als Abbild des Krieges zu verstehen, sondern als Modell zu begreifen, das von redaktionellen Strukturen wesentlich geprägt wird. “2 Aber obwohl die moderne Kriegsberichterstattung ohne den Journalismus nicht vorstellbar ist, stellt sich die Frage, ob der moderne Journalismus in jedem Krieg ein System sui generis ist, das die Regeln des eigenen Handelns selbst bestimmt. Der Mainstream der gegenwärtigen Journalismus-Forschung folgt dem systemtheoretischen Paradigma. Aber ist des-

1 Vgl. pars pro toto die Sonderhefte des European Journal of Communication 15. Jg./Nr. 3 (2000) und Medien \& Kommunikationswissenschaft 53. Jg./Nr. 2-3 (2005).

2 Löffelholz, Martin: Kriegsberichterstattung, in: Weischenberg, Siegfried/Kleinsteuber, Hans J./Pörksen, Bernhard (Hg.): Handbuch Journalismus und Medien, (Praktischer Journalismus, Bd. 60), Konstanz 2005, S. 181-185, Zit. S. 183. 
sen „Prinzip der journalistischen Autonomie bei der Kriegsberichterstattung “ 3 jenseits normativer Wünschbarkeit überhaupt gegeben? Was setzt ihr wann, wo und warum Grenzen?

Zunächst muss ein geeigneter Untersuchungsbereich mit genau definierten Randbedingungen bestimmt werden: Wenn nicht in einem Krieg mehrere Länder miteinander verglichen werden sollen und da kein Krieg historisch dem anderen gleicht, wird ein Längsschnitt nötig; nur so kann man zu Aussagen kommen, die nicht nur für den Einzelfall Gültigkeit besitzen. Zu diesem Zweck sollen die drei letzten großen Kriege, die Deutschland geführt hat, betrachtet werden: Der deutsch-französische Krieg von 1870/71 sowie der Erste und der Zweite Weltkrieg, 1914-1918 bzw. 1939-1945. Das setzt raum-zeitliche Randbedingungen: 1) die der politischen Systeme: Weder die Krieg führenden deutschen Staaten von 1870/71 noch das Kaiserreich des Ersten Weltkriegs und selbstredend erst recht nicht die NS-Diktatur gestatteten demokratische Pressefreiheiten; 2) die Art der Kriegsbeteiligung: Alle drei Kriege bedeuteten für Deutschland mehr oder minder existenzielle Krisen. Am Ende von Erstem und Zweitem Weltkrieg stand jeweils der Untergang der bestehenden politischen Ordnung, 1871 wurde eine neue begründet.

Für jeden der drei Kriege ist auf allen Ebenen Quellenmaterial überliefert: zum „propagandistischen“ Input, zur journalistischen Verarbeitung und zu den Wirkungen - wiewohl gleichzeitig auch für jede Stufe Quellenmaterial fehlt, häufig sogar äußerst wichtiges - Überlieferungsungleichgewichte sind in der kommunikationshistorischen Forschung die Regel. Zum propagandistischen Input gibt es eine Vielzahl an Untersuchungen ${ }^{4}$. Die Rezeption der Kriegsberichterstattung in der deutschen Öffentlichkeit ist andernorts detailliert untersucht worden und wird hier weitgehend ausgeblendet. ${ }^{5}$

Die wichtigsten zentralen Lenkungseinrichtungen waren im Ersten Weltkrieg das Kriegspresseamt und im Zweiten Weltkrieg das Ministerium für Volksaufklärung und Propaganda. Für den Krieg von 1870/71 könnte man, allerdings mit deutlichen Einschränkungen, die literarischen Bureaus nennen, insbesondere das preußische. Traditionell, schon seit der Frühen Neuzeit, wurde der Außenwirkung der Presseberichterstattung ein hoher Stellenwert zugesprochen. Daher beanspruchten die Außenministerien der deutschen Staaten, nach der Reichsgründung das Auswärtige Amt (AA), ein gewichtiges Wort in der Presselenkung. Bismarcks auswärtige Pressepolitik ist schon vor Jahrzehnten eingehend untersucht worden. ${ }^{6}$

Eine wichtige Instanz war das Militär. Das gilt selbst im Zweiten Weltkrieg und im Krieg von 1870/71, obwohl beide primär unter machtpolitischen Gesichtspunkten geführt wurden. Im Ersten Weltkrieg, in dem der militärische Apparat bis hin zur 3. Obersten Heeresleitung unter Hindenburg und Ludendorf immer stärker sein Primat

3 Lerg, Winfried B.: Geschichte der Kriegsberichterstattung. Ein Literaturbericht, in: Publizistik, 37/1992, Nr. 3, S. 405-422, hier: S. 406. Vgl. Richter, Simone: Journalisten zwischen den Fronten. Kriegsberichterstattung am Beispiel Jugoslawien, Wiesbaden 1999, S. 34.

4 U. a. Kohlmann-Viand, Doris: NS-Pressepolitik im Zweiten Weltkrieg. Die „vertraulichen Informationen“ als Mittel der Presselenkung, (Kommunikation und Politik, Bd. 23), München/ New York/London/Paris 1991. Koszyk, Kurt: Deutsche Pressepolitik im Ersten Weltkrieg, Düsseldorf 1968. Koszyk, Kurt: Erhard Deutelmoser - Offizier und Pressechef (1873-1956), in: Publizistik, 30/1985, S. 509-534.

5 Stöber, Rudolf: Die erfolgverführte Nation. Deutschlands öffentliche Stimmungen 1866 bis 1945, Stuttgart 1998.

6 Naujoks, Eberhard: Bismarcks auswärtige Pressepolitik und die Reichsgründung (1865-1871), Wiesbaden 1968. 
gegenüber der Politik durchzusetzen wusste, bestimmte das Militär sogar über die zentrale Lenkungsinstanz - das Kriegspresseamt. In den Kriegen waren also jeweils innenpolitisch, außenpolitisch und militärpolitisch agierende Institutionen mit Fragen der Kriegsberichterstattung, Pressepolitik und Propaganda befasst. Daneben existierte eine Vielzahl minder wichtiger Institutionen in der Etappe - von den Kreiskommandos bis zu den Bezirksregierungen.

Alle Institutionen machten der journalistischen Verarbeitung des kriegerischen Geschehens Vorgaben: einerseits dynamische, andererseits statische. Dynamisch waren beispielsweise die ständig aktualisierten Presseanweisungen der NS-Zeit oder die Sprachregelungen der Berliner Pressekonferenz des Ersten Weltkriegs. Halbwegs statisch waren z. B. die Zensurbücher des Ersten Weltkriegs.

Obwohl die Quellenüberlieferung vergleichsweise gut ist, gilt das für manche der zentralen Steuerungsinstrumente leider nicht; z. B. zu der in allen drei Kriegen jeweils wichtigsten deutschen Nachrichtenagentur: in den ersten beiden Kriegen „Wolff's Telegraphisches Bureau“ (WTB), in der NS-Zeit das „Deutsche Nachrichtenbüro“ (DNB). Das 1849 gegründete WTB stand seit 1869 unter dem Einfluss der preußischen Regierung, im Ersten Weltkrieg unter dem der Reichsregierung; das 1933 aus WTB und Hugenbergs Telegraphen-Union hervorgegangene DNB wurde vom Propagandaministerium gesteuert.? Immerhin sind im Bundesarchiv Berlin Teilbestände der DNB-Meldungen archiviert, allerdings erst ab dem Spätherbst 1944. ${ }^{8}$ Die Agenturmeldungen in den beiden früheren Kriegen können nur indirekt aus der Zeitungsberichterstattung erschlossen werden. ${ }^{9}$

Die eingangs gestellte modernistische Frage nach der journalistischen Autonomie lässt sich nicht direkt aus Quellen erschließen, die Auskunft zur Befolgung journalistischer Alltagsroutinen und damit zur Autonomie des journalistischen Systems unter der massiven Einflussnahme durch propagandistisch lenkende Institutionen geben. Nur z.T. kann auf Tagebücher zurückgegriffen werden. ${ }^{10}$ Die Journalistenbiographien wenden sich eher den allgemein- und zensurpolitischen Besonderheiten zu und geben zum Alltag nur Hinweise wie „fuhr ich zur Kochstraße und eröffnete um elf Uhr in Zivil die Redaktionskonferenz der DAZ. "11 Zudem werden Autobiographien immer pro domo geschrieben und sind nicht in allen Details verlässlich. ${ }^{12}$

Um der Fragestellung dennoch nachgehen zu können, bietet sich methodisch der indirekte Indizienbeweis an: die Auswertung der Medien selbst und ihr quellenkritischer Vergleich mit dem „propagandistischen“ Input. Die Methode der historischen Quellenkritik ist keineswegs mit ihrem Gegenteil, der normativen kritischen Theorie zu ver-

7 Basse, Dieter: Wolff‘s Telegraphisches Bureau 1849 bis 1933, (Kommunikation und Politik, Bd. 21), München/New York/London/Paris 1991. Wilke, Jürgen (Hg.): Telegraphenbüros und Nachrichtenagenturen in Deutschland. Untersuchungen zu ihrer Geschichte, (Kommunikation und Politik, Bd. 24), München/New York/London/Paris 1991.

8 BA Berlin, R 34 .

9 Wilke, Jürgen: Die Telegraphischen Depeschen des Wolff'schen Telegraphischen Büros (WTB), in: Publizistik, 49/2004, Nr. 2, S. 125-151.

10 Busch, Moritz: Tagebuchblätter, 3 Bde., Leipzig 1899. Sösemann, Bernd (Hg.): Theodor Wolff. Tagebücher 1914-1919. Der Erste Weltkrieg und die Entstehung der Weimarer Republik in Tagebüchern, Leitartikeln und Briefen des Chefredakteurs am „Berliner Tageblatt“ und Mitbegründers der „Deutschen Demokratischen Partei“, 2 Bde., Boppard 1984.

11 Z. B. Silex, Karl: SX. Mit Kommentar. Lebensbericht eines Journalisten, Frankfurt a. M. 1968, S. 212, zum Krieg: S. 211-226.

12 Vgl. Fn. 74. 
wechseln. Sie ist weder Inhaltsanalyse noch Hermeneutik, weder quantitative Auswertung von Stichproben einer gegebenen Grundgesamtheit noch selbstgenügsames Lesen der Texte. Zur historischen Quellenkritik gehört neben der äußeren und inneren Kritik (W-Fragen, Text- und Überlieferungssicherung, Faktenrichtigkeit anhand verlässlicher Parallelüberlieferung etc.) insbesondere die Berücksichtigung des Entstehungskontextes. Quellenkritik besteht zunächst einmal in der Prüfung der Belastbarkeit der Quellen. Die Angabe eines Samples darf durchaus fehlen. Um z. B. die Aussage absichern zu können, dass das „Berliner Tageblatt“ (BT) im Ersten Weltkrieg grundsätzlich keine OHL-Statistiken zum unbeschränkten U-Boot-Krieg verwendete, mussten die Zeitungsausgaben des fraglichen Zeitraums (ab Dezember 1916) vollständig durchgesehen werden.

Auf eine Inhaltsanalyse alleine zu setzen, wäre geradezu fahrlässig - daran ändert die Beachtung der Sauberkeit statistischer Verfahren nichts. Stichprobenziehung (ob Quota oder Random) können in die Irre führen, wie mit folgendem Vorgang erläutert werden soll: In einer Anfrage des Regierungspräsidenten von Oberbayern, Theodor von Zwehl, bei seinen Kollegen hieß es 1870: „Der Redacteur des dahier [in München] erscheinenden Blattes „das bayer. Vaterland“ hat beantragt, die k. Polizeidirektion München anzuweisen, bei künftig etwa für veranlaßt erachtete polizeiliche Beschlagnahmen des Blattes jedesmal die betreffende Stelle oder Artikel zu bezeichnen, damit er in die Lage komme, solche bei neuer Auflage der betreffenden Nummer hinweglassen zu können, u. hinzugefügt, andere Kreis-Regierungen hätten dieses bereits ausdrücklich befohlen. " Dem Ersuchen des dezidiert antipreußischen Journalisten Johann Baptist Sigl war ein Kleinkrieg vorausgegangen, der darin gipfelte, dass eine Zeitungsausgabe schließlich als unbedrucktes Papier erschienen war. ${ }^{14}$ Der Regierungspräsident von Oberfranken, Ernst Frhr. von Lerchenfeld, ließ nach München antworten: „Auf jenseitige Zuschrift vom 12. d. M. beehren wir uns zu erwidern, daß wir noch keinen Anlaß hatten, in der angedeuteten Richtung Anordnungen zu [er]lassen, übrigens keine Bedenken tragen würden, anzuordnen, daß bei Beschlagnahme den dasselbe veranlassenden Artikel dem Redacteur bekannt zu geben sey." ${ }^{15}$ Quellenkritisch folgt aus dem Vorgang, dass nicht nur in Systemen mit Vorzensur - wie im Deutschland des Ersten Weltkriegs -, sondern auch in solchen mit Nachzensur - wie im Bayern zwischen 1850 und 1874 - sich Konflikte nicht mehr aus den überlieferten Zeitungsbeständen rekonstruieren lassen: Im Zweifel enthalten diese nämlich die bereinigte Neuausgabe einer Zeitung. Im Übrigen steckt in dem Ansinnen des Redakteurs auch die implizite Andeutung, zur Selbstzensur bereit zu sein. Wenn man daraufhin die Berichterstattung kontrolliert, stellt man fest, dass die Selbstzensur auch in Oberfranken funktionierte. ${ }^{16}$

13 RegPr. Oberbayern an RegPr. Oberfranken, Schreiben vom 12.10.1870 (Tb-Nr. 33334), in: BaSTA, K3 F VIII, Nr. 15, Bd. I.

14 BamTb, Nr. 202 (26.7.1870), S. 1719.

15 RegPr. Oberfranken an RegPr. Oberbayern, Schreiben [Entwurf] vom 19.10.1870, in: Ebd.

16 Der mögliche Verdacht, der Bayreuther Oberpräsident habe mit seiner Antwort die Nachlässigkeit seiner Behörde kaschiert, bestätigte sich nicht. Zugleich lässt sich aber an folgendem Bericht zeigen, dass heutige Wertmaßstäbe sich nicht auf die damalige Kommunikationskontrolle - weder in Bayern noch Preußen - anwenden ließen: „Einem Spezialberichte der Frankf. Ztg. über die Schlacht bei Sedan am 1. Sept. entnehmen wir Folgendes: [...] Die Bestialität feierte schreckliche Orgien. Beim Rückzuge, zu dem die Bayern mehrmals gezwungen waren, wurden zurückgelassene Verwundete von den Weibern in die brennenden Häuser geworfen! Der Anblick solcher Greuel veranlaßte die Soldaten zu neuem Vorgehen. Kein Pardon ward gegeben. In die Häuser, von denen jedes eine Festung, wurden von Pionieren Feuerbrände geworfen. Das ganze Dorf ging in Flammen auf.“ In: BamTb, Nr. 246 (8.9.1870), S. 2059. 
Im konkreten Fall sind je Krieg mehrere Zeitungen untersucht worden. Wichtigstes Auswahlkriterium war die unterschiedliche Bewegungsfreiheit der Blätter. Für den Krieg 1870/71 wurden „Provinzial-Correspondenz“ (PC) und „Norddeutsche Allgemeine Zeitung“ (NAZ), für den Ersten Weltkrieg letztere und die Berliner Ausgabe des „Berliner Tageblatt“ (BT), für den Zweiten Weltkrieg die Berliner Ausgabe des „Völkischen Beobachter“ (VB) und die Reichsausgabe von „Der Angriff“ ausgewählt. PC und NAZ waren bismarck-offiziös, PC das „Zentralorgan“ für die preußische Kreisblattpresse ${ }^{17}$, die NAZ eine überregionale Qualitätszeitung. Das liberale BT unter seinem Chefredakteur Theodor Wolff war im Ersten Weltkrieg eine der unabhängigsten Zeitungen. Der VB besaß durch seinen Chefredakteur Alfred Rosenberg eine gewisse Unabhängigkeit gegenüber Goebbels, der 1928 den „Angriff“ gegründet, ihn allerdings 1933 an Robert Leys Pseudogewerkschaft „Deutsche Arbeitsfront“ abgetreten hatte. Ein verbreiteter Irrtum spricht dabei selbst für die NS-Zeit einigen ehemaligen Qualitätszeitungen - u. a. „Deutsche Allgemeine Zeitung“ oder „Frankfurter Zeitung“ - die größeren Freiheiten zu. In der NS-Zeit aber gilt, dass gerade diese besonders kritisch betrachtet wurden. Überzeugt nationalsozialistische Zeitungen, hinter denen wichtige NS-Führer standen, waren in ihrer Autonomie hingegen weniger eingeschränkt. Zwei unterschiedliche Regionalzeitungen, das konservative „Bamberger Tagblatt“ (BamTb) und das katholische „Bamberger Volksblatt“ (BamVb) ergänzen das untersuchte Medienangebot.

Die Medieninhalte sollen auf die Frage untersucht werden, wie es um die Erfüllung journalistischer Funktionen stand. Latente Funktionen wie Sozialisations-, Integrationsoder Rekreationsfunktion tragen selbstredend wenig zum Thema bei. Aussagekräftig im Sinne der Fragestellung scheinen hingegen die Informations- und Meinungsbildungsfunktionen zu sein; so uneinig die Literatur sonst in der Benennung der massenmedialen Funktionen ist, sie werden immer zu den wichtigsten gezählt. Allerdings fällt die Abgrenzung zwischen Information und Meinungsbildung nicht leicht, weil Informationen immer Meinungsbildung unterstützen und Meinungen umgekehrt immer auch Informationen liefern. Als dritte Funktion wurde die seltener genannte Orientierungsfunktion ausgewählt; sie spielte schon am Beginn der Zeitungsgeschichte, die eng mit Kriegsberichterstattung verknüpft war, eine zentrale Rolle: Im Dreißigjährigen Krieg setzten sich die Avisen und Relationen gegen andere Periodika nicht zuletzt deshalb durch, weil sie „defensive Orientierung“, wie es der Pressehistoriker Johannes Weber ausgedrückt hat, boten. ${ }^{18}$

\section{B. Krieg und Politik, Kriegsberichterstattung und politische Kommunikation}

Nach dem Methodischen sind noch einige Begriffe zu definieren: insbesondere politische Kommunikation, Propaganda und Kriegsberichterstattung. Doch wird man deren Verhältnis nicht analysieren können, wenn nicht zuvor auch das Verhältnis von Krieg und Politik geklärt ist.

Wer Krieg und Politik bestimmen will, landet zwangsläufig bei Carl von Clausewitz

17 Die PC wird in einem DFG-Projekt derzeit für breite Nutzerschichten aufbereitet, vgl. http:// amtspresse.staatsbibliothek-berlin.de/index.html.

18 Weber, Johannes: Deutsche Presse im Zeitalter des Barock. Zur Vorgeschichte des öffentlichen politischen Räsonnements, in: Jäger, Hans-Wolf (Hg.): „Öffentlichkeit“ im 18. Jahrhundert, (Das 18. Jahrhundert. Supplementa, Bd. 4), Göttingen 1997, S. 137-149, hier S. 144. 
und Carl Schmitt. Schmitt hatte Politik als Bürgerkrieg definiert. ${ }^{19}$ Er lehnte die auf Interessenausgleich bedachte Demokratie vehement ab und definierte Politik als emotional aufgeladenen Kampf um Leben und Tod, anstatt Interessen- und mithin Sachpolitik als Kriterium des Politischen zu benennen. Clausewitz hingegen hatte - obwohl häufig das Gegenteil zu lesen ist - den Krieg der Politik untergeordnet. ${ }^{20}$ Er bestritt, anders als Schmitt, dass der Krieg (und erst recht die Politik) dem Manichäismus von Freund und Feind gehorche, und definierte Politik (und somit auch Kriege) primär über Interessen. Krieg und Politik sollen mit Clausewitz und gegen Schmitt wie folgt bestimmt werden:

Politikfähig, Gegenstand der Politik, ist alles, was sich artikulierbaren Interessen zuordnen lässt. Im weitesten Sinne räumt das auch persönlichen Interessen Platz ein, im engeren sollen aber nur überindividuelle, aggregierte Interessen komplexer Sozialsysteme (von privaten Institutionen, Organisationen, Verbänden über Nationen bis zu supranationalen Institutionen) als politikfähig betrachtet werden. ${ }^{21}$ Kriege sind eines der Mittel zur Durchsetzung dieser Interessen, setzen aber auf staatlich organisierte militärische Gewalt.

Ebenso wie der Krieg der Politik unterzuordnen ist, verhält es sich mit politischer Kommunikation; sie kommuniziert Politik und ist dieser zugeordnet. Otfried Jarren und Patrick Donges haben sie in ihrer Einführung wie folgt definiert: „Politische Kommunikation ist der zentrale Mechanismus bei der Formulierung, Aggregation, Herstellung und Durchsetzung kollektiv bindender Entscheidungen. Insofern ist politische Kommunikation nicht nur Mittel der Politik. Sie ist selbst auch Politik. "22 Ergänzt man diese Definition um den Kernbegriff „Öffentlichkeit“, so ist die Arbeitsdefinition zugleich von Diplomatie abgegrenzt. Mit Clausewitz wird analog zum Krieg als Instrument der Politik die Kriegsberichterstattung zur Ausprägung der politischen Kommunikation.

Dabei ist politische Kommunikation ein Oberbegriff zu politischer PR, Propaganda und Kriegsberichterstattung. Dass die Kriegsberichterstattung nicht trennscharf von den anderen Ausprägungen der politischen Kommunikation zu unterscheiden ist, liegt primär an ihren vielgestaltigen Formen. Kriegsberichterstattung besteht, wie anderer Journalismus auch, aus Informationen über Tatbestände und aus deren Bewertung. Meinung und Nachricht lassen sich jedoch kaum trennen, weil die verschwiegene Nachricht mindestens so wichtig wie die berichtete ist, und beide zusammen Bewertungen implizieren. Diese wiederum reichen von den Urteilen über kleinere Begebenheiten des Krieges bis hin zu den großen strategischen Fragen von Kriegsziel und -zweck.

Kriegsberichterstattung ist aber noch mehr: Im engeren Sinne ist sie informative und wertende Berichterstattung über die militärischen Ereignisse des Krieges; im weiteren auch psychologische Kriegsführung. Im weitesten Sinne ist sie Berichterstattung im Krieg. ${ }^{23}$ Auf den ersten Blick erscheint das als Entgrenzung des Begriffs, die jede weitere Analyse unmöglich macht. Schon die Berichterstattung über Lebensmittelversorgung

19 Schmitt, Carl: Der Begriff des Politischen. Text von 1932 mit einem Vorwort und drei Corollarien, 5. Nachdr. der 7. Aufl., München/Berlin 2002, S. $26 f$.

20 Clausewitz, Carl von: Vom Kriege (Nachdruck der 1. Auflage von 1832-34), Augsburg 1990, S. 674-681 (VIII. Buch, Kap 6 B).

21 Vgl. Alemann, Ulrich von: Politikbegriffe, in: Nohlen, Dieter (Hg.): Lexikon der Politik, 7 Bde., München 1992, Bd. 2, S. 298-301.

22 Jarren, Otfried/Donges, Patrick: Politische Kommunikation in der Mediengesellschaft. Eine Einführung. Band 1: Verständnis, Rahmen und Strukturen, (Studienbücher zur Kommunikations- und Medienwissenschaft, Bd. 1/2), 2 Bde., Wiesbaden 2002, S. 22.

23 Löffelholz (Fn. 2), S. 181. 
und -preise verdeutlicht jedoch, dass eine weite Perspektive der Realität im Krieg besser gerecht wird als eine einseitige Beschränkung auf die Berichterstattung von militärischen Dingen. Hitler und Goebbels, beide Zeitgenossen des Ersten Weltkriegs, fürchteten nichts so sehr wie eine Wiederholung der Geschichte. Daher legte die nationalsozialistische Presseberichterstattung im Allgemeinen großen Wert auf eine Betonung der Unterschiede, und im Besonderen wurde in den NS-Medien bei geeigneter Gelegenheit die gute Lebensmittelversorgung der Deutschen hervorgehoben. ${ }^{24}$ Kriegsberichterstattung ist demnach nicht nur Begleiterscheinung des Krieges, sie ist zugleich Teil der politischen Kriegsführung und somit ein Sonderfall politischer Kommunikation. Es macht wenig Sinn, sie auf die Berichterstattung über das militärische Geschehen zu begrenzen: Etliche Aspekte der Berichterstattung im Kriege ergeben erst in der Zusammenschau einen Gesamteindruck von der allgemeinen Kriegslage. Kriegsberichterstattung soll hier also als Teil der politischen Kommunikation in Kriegszeiten verstanden werden.

Im Unterschied zu den anderen Begriffen fällt die Definition von Propaganda schwer, da der Begriff durch seine Geschichte selbst kompromittiert wurde. Am sinnvollsten wäre eine begriffshistorische Annäherung; dies nähme zu viel Raum ein, darum sei auf die stimmigste verwiesen. ${ }^{25}$ Eine universal gültige Definition des kontaminierten Begriffs ist unmöglich. Hier soll Propaganda als Sonderform politischer Kommunikation verstanden werden, bei der die politischen Interessen eines zentralen Kommunikators einem heterogenen Adressatenkreis kommuniziert werden. Nimmt man Information, Meinungsbildung und Orientierung als wichtigste Funktionen des Journalismus, so vermittelt der Kommunikator mit Propaganda v. a. ihm genehme und nützliche Informationen und Meinungen und bietet den Adressaten zweckorientiertes Orientierungswissen an. Propaganda ist mithin eine Form persuasiver Kommunikation. ${ }^{26}$ Das in der Literatur bisweilen anzutreffende normativ-negative Verständnis von Propaganda, sie konstituiere Desinformation und -orientierung sowie eher überredende als überzeugende Meinungsbildung, scheint für die Analyse der Kriegsberichterstattung wenig hilfreich. Auch die prima vista einleuchtende Bemerkung, Propaganda, insbesondere die nationalsozialistische, gehe mit Gewaltandrohung einher, soll ausgeklammert bleiben, da die Gewaltandrohung nicht in den konkreten Kommunikaten, sondern in der Struktur des politischen und kommunikativen Systems angelegt war. Unnachahmlich rabulistisch hat es Joseph Goebbels formuliert: „Wir -, wir Propagandisten, wir wenden

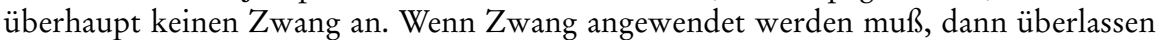
wir das anderen. Wir tuen das nicht, das ist Aufgabe von anderen Ressorts. “27

24 Boelcke, Willi A. (Hg.): „Wollt ihr den totalen Krieg?“ Die geheimen Goebbels-Konferenzen 1939-1943, München 1989, S. 280 (15.9.1942).

25 Schieder, Wolfgang/Dipper, Christof: Propaganda, in: Brunner, Otto/Conze, Werner/Koselleck, Reinhart (Hg.): Geschichtliche Grundbegriffe. Historisches Lexikon zur politisch-sozialen Sprache in Deutschland, 8 Bde., Stuttgart 1972-1997, Bd. 5, S. 69-112.

26 Merten, Klaus: Struktur und Funktion von Propaganda, in: Publizistik, 45/2000, Nr. 2, S. 143 162, hier: S. 151.

27 Heiber, Helmut (Hg.): Goebbels Reden. 1932-1945, 2 Bde., Augsburg 1991, Bd. 1, S. $247 f$. 


\section{Kriegsberichterstattung zwischen Information und Verschleierung, Meinungsbildung, Orientierung und Propaganda}

\section{A. Die Informationsfunktion}

Die Informationsfunktion war die erste und ist bis heute die wichtigste Funktion periodischer Medien. Zugleich ist sie aus militärischer Warte heikel, weil jede Information dem Feind dienen kann. Im deutsch-französischen Krieg wurde demgemäß erstens sehr zurückhaltend, zweitens meist mit Verspätung von bis zu drei Tagen berichtet. Das galt selbst für Zentralereignisse wie den Sieg bei Sedan am 1.9.1870: „Auf dem Schlachtfelde

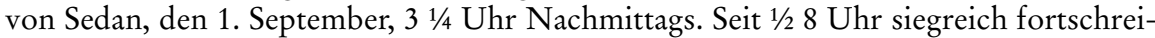
tende Schlacht rund um Sedan. - Garde, 4., 5., 11., 12. Corps und Baiern - Feind fast ganz in die Stadt zurückgeworfen. gez. Wilhelm [...] In der obigen Depesche heißt es ,siegreich fortschreitende Schlacht rund um Sedan' und diese wenigen Worte geben über die Hartnäckigkeit und lange Dauer der Schlacht vollständige Auskunft. “28

Insgesamt, so lässt sich aus der Harmlosigkeit der Presseberichterstattung und aus Akten der Innenministerien und den nachgeordneten Behörden schließen, war die Selbstzensur wohl zu groß und der Krieg zu kurz und zu erfolgreich, als dass es viele offene Konflikte hätte geben können. Nur in krassen Fällen, z. B. Falschmeldungen und Desinformationen, sind sie noch aus den Akten rekonstruierbar. In solch einem Fall schärfte das bayerische Staatsministerium des Innern ein: „Die Preßpolizeibehörden sind deshalb neuerdings anzuweisen, bei Zuwiderhandlung gegen die Vorschriften, welche für die Veröffentlichung von auf den Krieg bezüglicher Nachrichten maßgebend sind, unnachsichtlich die Beschlagnahme zu verfügen. “29 Diese strenge Linie galt von Anfang an und die Zeitungen bemühten sich zumeist sowohl darum, die behördlichen Vorgaben zu kommunizieren, sowie die eigene Zurückhaltung als patriotische Pflicht darzustellen. ${ }^{30}$

Im Ersten Weltkrieg resümierte ein Autor des „Berliner Tageblatt“ (BT): „Schon im Krieg von 1870 wurde die Kriegsberichterstattung auf deutscher Seite viel strenger überwacht, als es bisher üblich war. Die deutsche Heeresleitung ging von dem Grundsatze aus, daß die Presse vor den eigentlich entscheidenden Schlägen ebenso gute Dienste durch Schweigsamkeit leisten müsse, wie nach getaner Arbeit durch stimmungsvolle Berichte. ${ }^{31}$ Allerdings galt diese Maxime in keinem der drei Kriege uneingeschränkt: In beiden Weltkriegen gab es „stimmungsvolle“ Berichte, d. h. Reportagen, und vollständige Informationen nur nach erfolgreichen Schlachten; nach Niederlagen galt weiterhin Schweigen als erste Journalistenpflicht. Im deutsch-französischen Krieg war über solche Rückschläge noch nicht zu berichten gewesen. Im Ersten wie im Zweiten Weltkrieg

28 NAZ 9. Jg./Nr. 204 (3.9.1870), S. 1. Vgl. Welch ein ergreifender Augenblick!, in: PC 8. Jg./Nr. 36 (7.9.1870), S. 1. Vgl. BamTb Nr. 241 (3.9.1870), S. 1.

29 Die erwähnten Bestimmungen sind die Art. 119 und 120 BayerStGB sowie die Verordnung vom 17.7.1870, Reg.Bl. 1870, Nr. 48. Staatsministerium des Innern an Regierungen, Runderlass vom 30.11.1870, in: BaSTA, K3 F VIII, Nr. 15, Bd. I. Im Dezember/Januar 1870/71 folgen noch weitere Beschlagnahme-Rescripte, die insgesamt drei namentlich genannte und einige ungenannte und nicht quantifizierte Vorgänge betreffen. Vgl. ebd.

30 „Durch k. Verordnung wird der Presse jede Mittheilung über militärische Operationen verboten.“ BamTb, Nr. 195 (19.7.1870), S. 1663. „Es ist selbstverständlich, daß bei jetziger Sachlage über Truppenzüge und Dislocationen keine Veröffentlichung gemacht werden kann.“ BamTb, Nr. 193 (17.7.1870), S. 1647.

31 [Prof. Dr.] v. Heigel: Krieg und Presse, in: BT 43. Jg./Nr. 464 (12.9.1914), S. 1f., hier: S. 1. 
wurden Niederlagen entweder komplett verschwiegen oder erst nach einiger Zeit in der Berichterstattung hinter dürren Worten der amtlichen Depeschen versteckt; bisweilen wurden sie gar als Erfolge - zumeist mit statistischem Material untermauert - ausgegeben. Dafür lassen sich etliche Beispiele anführen, ob Abbruch der Marne-Schlacht 1914, Verdun 1916, Michaels-Offensive 1918, der Dies Ater der deutschen Front am 8.8.1918, die Schlacht am Kursker Bogen 1943, die Ardennen-Offensive 1944/45 und andere mehr. So manches Desaster wurde mythisch überhöht: „Westlich Langemarck drangen junge Regimenter unter dem Gesange ,Deutschland, Deutschland über alles' gegen die erste Linie der feindlichen Stellungen vor und nahmen sie. Etwa 2.000 Mann französischer Linieninfanterie wurden gefangen und sechs Maschinengewehre erbeutet. ${ }^{\text {" }} 2$ Dass dabei allein auf deutscher Seite ca. 2.000 Soldaten starben, wurde in der Meldung, die fast die gesamte deutsche Presse brachte, verschwiegen. ${ }^{33}$ Stalingrad wäre, den „Endsieg“ vorausgesetzt, sicher ebenfalls mythisiert worden. Die Voraussetzungen wurden mit der Berichterstattung zum Untergang der 6 . Armee gelegt. ${ }^{34}$

Insbesondere die auf den ersten Blick wohlfeilen statistischen Angaben bargen Probleme. Prima Vista erfüllen die Medien mit Statistiken ihre Informationsfunktion am besten, weil sie vage Tendenzaussagen (,immer mehr“ oder „immer weniger“) anscheinend mit Substanz füllen. Doch liest man die Presse der drei Kriege aufmerksam, so fällt v. a. auf, dass sie nicht das bedeuten, was sie explizit sagen. Z. T. deutet die Bekanntgabe eigener Verluste auf einen deutschen Sieg, z. T. die gegnerischer Verluste auf eine deutsche Niederlage; dann wieder sind die Zahlen geschönt, oder manchmal auch korrekt - sowohl in Inhalt wie in Kontext. Im Krieg von 1870/71 wurden Zahlen zu eigenen Verlusten, Namen oder Todesanzeigen erst nach den frühen Siegen erwähnt. ${ }^{35} \mathrm{Im}$ Ersten Weltkrieg, nach der ersten entscheidenden Niederlage in der Marne-Schlacht (5.12.9.1914), meldete eine WTB-Meldung die Gefangennahme von 40.000 Franzosen. ${ }^{36}$ Zwei Tage später hieß es im BT vorsichtiger: „Das Ringen an der Marne. Abwarten! ${ }^{\text {(37 } 7 n}$ der NAZ war der Stopp des deutschen Vormarschs explizit als Erfolg gepriesen worden: Deutsche Truppen hielten den "gewaltig überlegenen Angriff [der Alliierten] mit solcher Unerschütterlichkeit aus, daß nicht nur unsere Kolonnen in voller Ruhe zurückgenommen werden konnten, sondern auch der feindliche Vorstoß blutig zusammenbrach.“"38 Implizit wurde damit allerdings der Misserfolg angedeutet. Als sich die letzte deutsche Großoffensive des Ersten Weltkriegs, die „Michael-Offensive“, festgelaufen hatte und kurz vor dem Abbruch stand, machte das BT mit der Erfolgsmeldung auf: „Seit 21. März

32 WTB: Amtlich, in: BT 43. Jg./Nr. 575 (11.11.1914), S. 1. Wortgleich in: NAZ 54. Jg./Nr. 280 (12.11.1914), S. 1; BamTb 80. Jg./Nr. 274 (12.11.1914), S. 1; BamVb 42. Jg./Nr. 265 (12.11.1914), S. 1.

33 Vgl. Unruh, Karl: Langemarck: Legende und Wirklichkeit, Koblenz 1986.

34 Vgl. u. a. Rosenberg, Alfred: Die Helden der 6. Armee, in: VB 56. Jg./Nr. 35 (4.2.1943), S. $1 f$. Der heroische Endkampf in Stalingrad, in: BamTb Nr. 27 (2.2.1943) S. 1.

35 Am 13. August 1870, nach der Schlacht von Weißenburg, wurden die ersten Bamberger Toten namentlich genannt, in der gleichen Ausgabe auch die erste Todesanzeige. Vgl. Telegramm. Bayerische Verlustliste der 3 Bataillone des 5. Inf.-Reg. bei der Einnahme von Weißenburg, in: BamTb, Nr. 220 (13.8.1870), S. 1856f. Todesanzeige Ludwig Mühlbauer, in: Ebd., S. 1859.

36 WTB: Noch viel mehr Kriegsgefangene, in: BT 43. Jg./Nr. 465 (13.9.1914), S. 1.

37 Das Ringen an der Marne. Abwarten!, in: BT 43. Jg./Nr. 469 (15.9.1914), S. 1. Vgl. Nerven behalten, in: BamVb 42. Jg./Nr. 213 (14.9.1914), S. 1.

38 Kämpfe um Paris, in: NAZ 53. Jg./Nr. 220 (13.9.1914), S. 1. Vgl. Günstige Lage in Ost und West. Verheißungsvolle Nachricht aus Paris, in: BamTb 80. Jg./Nr. 215 (14.9.1914), S. 1. 
191.454 Gefangene“. ${ }^{39}$ Einerseits könnte man die Zahl als journalistische Routine interpretieren: Zum Quartalswechsel bot sie Gelegenheit, Bilanz zu ziehen. Doch lag hier eher die „Bestechung“ der Redaktion mit dem Appell an die journalistischen Informationsroutinen durch das Kriegspresseamt vor - ganz im Sinne der Determinismusthese, deren Gültigkeit zumindest für wichtige politische Themen und Kommunikatoren cum grano salis unbestritten ist. ${ }^{40}$

Einen ähnlichen Umgang mit Niederlagen, die sich durch Statistiken in Siege umdeuten ließen, brachte der Zweite Weltkrieg in großer Zahl. Die „Michael-Offensive“ ist vergleichbar mit dem Unternehmen „Zitadelle“, der Schlacht am Kursker Bogen vom 5.-13.7.1943; hier ging die Wehrmacht letztmalig an der Ostfront in die Offensive, musste sie aber schließlich abbrechen. Die Offensive wurde von der Kriegsberichterstattung zunächst verhalten aufgegriffen. Bis zum 7.7. fehlte jeder Hinweis, erst am 8.7. bestimmte die Schlacht in VB und „Angriff“ fast wortgleich den Aufmacher, nur die Auszeichnung unterschied sich. ${ }^{41}$ Ein DNB-Artikel über den „Verlauf der Kämpfe“ berichtete im VB relativ vorsichtig. ${ }^{42}$ Die Berichte über hohe Feindverluste setzen sich in den nächsten Tagen fort, aber ausgerechnet am 13.7., als das OKW die Schlacht abbrach, titelten VB und „Angriff“ fast synchron mit Erfolgen. ${ }^{43}$ Im VB hieß es u. a.: „Auch keine der großen Kesselschlachten der Jahre 1941 und 1942 brachte derartige hohe Abschlußzahlen in so kurzer Zeit und auf so kurzem Raum. “44 Zahlen zu deutschen Verlusten fehlten gänzlich - wie in früheren Berichten auch. Beinahe täglich wurden steigende sowjetische Verluste hochgeschrieben: bis zum 22.7.1943 im VB auf 5.500, im „Angriff“ auf 4.827 Panzer. ${ }^{45}$ Die tatsächlichen Abschlüsse lagen mindestens um die Hälfte unter den publizierten. ${ }^{46}$ Nach dreitägigem Schweigen gestand der VB erstmalig eine sowjetische Sommeroffensive ein; im „Angriff“ wurden die sowjetischen Verluststatistiken noch in den nächsten Tagen fortgeschrieben, dass die Initiative aber inzwischen bei der Roten Armee lag, machte der Aufmacher, ebenfalls am 25.7., deutlich. ${ }^{47}$

Aber nicht nur in der Niederlage, auch im Erfolg wirkten die pseudoobjektiven sta-

39 Seit 21. März 191.454 Gefangene, in: BT 47. Jg./Nr. 331 (1.7.1918), S. 1.

40 Vgl. Baerns, Barbara: Öffentlichkeitsarbeit oder Journalismus? Zum Einfluß im Mediensystem, (Bibliothek Wissenschaft und Politik, Bd. 32), Köln 1985.

41 Die Schlacht zwischen Orel und Bjelgorod. In zwei Tagen: 637 Flugzeuge, 300 Panzer der Sowjets abgeschossen, in: VB 56. Jg./Nr. 189 (8.7.1943), S. 1. Feindangriff abgewehrt / Einbruch in Sowjetstellungen. Sowjets verloren 300 Panzer und 637 Kampfflugzeuge, in: Der Angriff Nr. 165 (8.7.1943), S. 1.

42 Der Verlauf der Kämpfe, in: VB 56. Jg./Nr. 189 (8.7.1943), S. 1.

43 Hohe Beutezahlen aus der Ostschlacht. [...] Wie zu Beginn des Ostfeldzugs. Sowjets verloren 28.000 Gefangene[,] 1640 Panzer und 1400 Geschütze, in: VB 56. Jg./Nr. 194 (13.7.1943), S. 1. Ostfront: 28.000 Gefangene und 1640 Panzer vernichtet, in: Der Angriff Nr. 169 (13.7.1943), S. 1.

44 VB 56. Jg./Nr. 194 (13.7.1943), S. 1.

45 Über 5.500 Panzer in 16 Tagen, in: VB 56. Jg./Nr. 203 (22.7.1943), S. 1. 4.827 Panzer, 2.201 Geschütze, 344 Flugzeuge, 45.172 Gefangene, in: Der Angriff Nr. 177 (22.7.1943), S. 1.

46 Schramm, Percy Ernst (Hg.): Kriegstagebuch des Oberkommandos der Wehrmacht, 8 Bde., München 1982, Bd. 6 [III.2] S. 748-817 (5.7.-21.7.1943). Das Kriegstagebuch verzeichnete nicht jeden Tag „Erfolgszahlen“, die Maximalangabe in dem Zeitraum belief sich auf 163 zerstörte Panzer. Ebd., S. 775 (13.7.1943).

47 Anhaltende schwere Abwehrkämpfe an den Brennpunkten der sowjetischen Sommeroffensive, in: VB 56. Jg./Nr. 206 (25.7.1943), S. 1. Alle Durchbruchversuche der Sowjets restlos gescheitert, in: Der Angriff Nr. 180 (25.7.1943), S. 1. Schwerpunkte der Angriffe wieder bei Orel, in: BamTb Nr. 173 (27.7.1943), S. 1. 
tistischen Zahlen ambivalent. Im Ersten Weltkrieg wurde v. a. im U-Boot-Krieg mit Statistiken operiert. Das hatte mehrere Gründe. Zum einen knüpfte sich an die Blockade der britischen Inseln durch deutsche U-Boote die Hoffnung, England werde den Kampf einstellen. Zum anderen war der U-Boot-Krieg wegen seiner möglichen Auswirkungen - der Kriegseintritt der USA erfolgte 1917 wegen der deutschen Angriffe auf die Schifffahrt - innenpolitisch höchst umstritten. Zum Dritten hatten die Briten eine Blockade über den Kontinent verhängt, infolgedessen wurde die deutsche Nahrungsmittelversorgung im Kriegsverlauf immer schlechter; da weckte jeder Bericht über versenkte Tonnage Genugtuung über die gelungene Vergeltung. Während aber die offiziöse NAZ nach der Verkündung des unbeschränkten U-Boot-Kriegs im Hungerwinter 1916/17 jede Schiffsversenkung meldete, brachte das BT, Gegner des U-Boot-Kriegs, keine dieser „Erfolgsmeldungen“, nicht einmal in einem Artikel, in dem man wegen der journalistischen Regel, griffige Erklärungen zu bieten, hätte erwarten dürfen, dass die deutschen U-Boote und die versenkte Tonnage genannt würden. ${ }^{48}$

Im Zweiten Weltkrieg wurden Statistiken häufig eingesetzt. Überschriften strichen regelmäßig die Feindverluste heraus. ${ }^{49}$ Eigene Verluste fehlten entweder ganz - das war die Regel - oder es wurden nur äußerst geringe zugegeben. Wo die Meldungen nur dazu dienten, offenkundig Schreckensnachrichten wie einen schweren Luftangriff abzupuffern, war die propagandistische Strategie schon auf den ersten Blick durchsichtig. Ein Beispiel bieten die bis Dresden schwersten anglo-amerikanischen Luftangriffe, die unter dem Operationsnamen „Gomorrha“ vom 25.7.-30.7.1943 gegen Hamburg geflogen wurden. In ihnen kamen mehr als 30.000 Menschen ums Leben; weite Teile des Hamburger Hafens und der Innenstadt gingen in Flammen auf. ${ }^{50}$ „Angriff“ und VB schwiegen zu den ersten Angriffstagen; erstmalig am 27.7. gab ein OKW-Bericht in dürren Worten von dem Angriff Kenntnis: „Das Oberkommando der Wehrmacht gibt bekannt: [...] Feindliche Fliegerverbände führten neue schwere Terrorangriffe gegen deutsche Städte. Am gestrigen Tage wurden Hamburg und Kiel sowie einige Orte im Norddeutschen Küstenraum [...] bombardiert. Dabei entstanden wieder schwere Verluste unter der Bevölkerung und starke Verwüstungen in den Wohngebieten der angegriffenen Städte. Luftverteidigungskräfte schossen aus den britisch-amerikanischen Angriffsverbänden 61 Flugzeuge ab. Fünf eigene Jagdflugzeuge gingen verloren. ${ }^{\text {"51 }}$ Das Kriegstagebuch des OKW hatte nur 48 Flugzeugabschüsse verzeichnet. ${ }^{52}$

Diese Presseangaben konnte niemand überprüfen. Zumindest Goebbels war das durchaus bewusst: Er meinte, im Luftkrieg ließe sich leichter lügen als im Landkrieg,

48 Englands Getreideversorgung, in: BT 46. Jg./Nr. 18 (11.1.1917), 2. Beiblatt, S. 1. Vgl. Sösemann (Fn. 10), passim, insb. S. 469-484 (1.1.-28.2.1917).

49 Wieder 37 Terrorbomber bei Angriff auf Köln abgeschossen. Insgesamt 90 Flugzeuge vernichtet, in: VB 56. Jg./Nr. 186 (5.7.1943), S. 1. U-Boote versenkten im Mittelmeer 21.000 BRT. 105 Feindflugzeuge abgeschossen, in: VB 56. Jg./Nr. 187 (6.7.1943), S. 1. Einzigartige Leistung der Luftverteidigung: 121 Bomber wurden zu „Fliegenden Särgen“, in: BamTb Nr. 243 (16./17.10.1943), S. 1. In fünf Kampftagen 495 Maschinen vernichtet, in: Ebd. Nr. 48 (26./27.2.1944) S. 1. u. v. m.

50 Außerdem wurden 277.330 Wohnhäuser völlig zerstört, 2.632 gewerbliche Betriebe, 580 Industriebetriebe, 80 Wehrmachtsanlagen, 24 Krankenhäuser, 277 Schulen und 58 Kirchen. Overesch, Manfred: Das III. Reich. Eine Tageschronik der Politik, Wirtschaft, Kultur, 2 Bde., Düsseldorf 1982, Bd. 2, S. 391.

5161 Terrorbomber abgeschossen, in: VB 56. Jg./Nr. 208 (27.7.1943), S. 1. Erneut 130 Panzer vernichtet. 61 Abschüsse bei Terrorangriff, in: Der Angriff Nr. 181 (27.7.1943), S. 1.

52 Schramm (Fn. 46), S. 836 (26.7.1943). 
da sich der geänderte Frontverlauf auf die Dauer nicht verschleiern lasse..$^{53}$ Aber selbst dort, wo die Meldungen in etwa der Realität entsprachen, kamen dem Publikum auf die Dauer Zweifel. Je mehr Panzer zerschossen und Flugzeuge zerstört, je monotoner Zahlen erbeuteter Geschütze repliziert wurden, desto stärker spekulierte man über das unerschöpfliche Material der Russen. Woher konnten denn die Sowjets immer noch neue Truppen ins Gefecht schicken ${ }^{54}$ Die Skepsis des Publikums galt sinngemäß für alle Zahlenangaben: Flugzeugabschüsse, Schiffsversenkungen, Panzer- und Geschützvernichtung, Gefangennahmen.

In welchem Ausmaß die „Polyphonie“ in den Durchhalteparolen des letzten Kriegsjahres zum dünnen Firnis journalistischer Pseudoautonomie wurde, beleuchtet ein Beispiel vom Dezember 1944. Am 16.12. waren deutsche Truppen im Westen zu ihrer letzten überraschenden Offensive angetreten - der „Ardennen-Offensive“. Ab dem 19.12.1944 berichteten „Angriff“ und VB in großer Aufmachung davon..$^{55}$ Am 20.12.1944 lautete die Tagesparole: „Das Fortschreiten der deutschen Offensive im Westen ermöglicht nunmehr eine Kontrastierung der tatsächlichen Kriegslage im Rahmen des OKW-Berichtes mit den grossmäuligen Voraussagen des Gegners. Ferner liegt gutes Material vor, das Staunen, Furcht und Bewunderung im Ausland über die Tatsache des deutschen Schlages widerspiegelt und, soweit es über DNB ausgegeben wird, im Rahmen der Kommentaranweisung von gestern wiederzugeben ist. " ${ }^{\circ 6}$ Anweisungsgetreu stellten VB und "Angriff“ am Folgetag OKW-Meldungen neben „Feindstimmen“ mit einem marginalen Unterschied heraus - im „Angriff“ nebeneinander, im VB untereinander. ${ }^{57}$

\section{B. Meinungsbildungsfunktion}

Die Meinungsbildung kann am detailliertesten oder am knappsten von den journalistischen Funktionen behandelt werden. Wenn alle anderen journalistischen Funktionen als der Meinungsbildung dienlich aufgefasst werden, könnte jeder Teilaspekt der Kriegsberichterstattung hierunter fallen. Um hier ein Ausufern zu verhindern, soll das wichtigste Thema der Meinungsbildung betrachtet werden: die Kriegsziele. Dabei lässt sich über die drei deutschen Kriege eine deutliche Abnahme der journalistischen Autonomie feststellen. In dem Krieg von 1870/71 bot die Presse schon infolge des Bündnissystems souveräner Staaten - der Norddeutsche Bund unter preußischer Führung und die süddeutschen Alliierten - eine im Ansatz durchaus heterogene Diskussion. Dass der Krieg, so er denn erfolgreich verlaufen sollte, automatisch auf eine deutsche Einigung hinauslaufen solle, war umstritten. Auch die Behandlung des geschlagenen Frankreich wurde

53 Boelcke (Fn. 24), S. 87f. (9.8.1940).

54 Boberach, Heinz (Hg.): Meldungen aus dem Reich 1938-1945. Die geheimen Lageberichte des Sicherheitsdienstes der SS, 17 Bde. und Registerband, Herrsching 1984, S. 2747 (11.9.1941); S. 3152 (12.1.1942).

55 Deutscher Angriff im Westen. Starke deutsche Kräfte angetreten. Vordere USA-Stellungen überrannt, in: Der Angriff Nr. 307 (19.12.1944), S. 1. Deutsche Offensive im Westen. In breiter Front: Stoß aus dem Westwall, Die USA-Stellungen zwischen Hohem Venn und Nordteil Luxemburgs überrannt, in: VB 57. Jg./Nr. 340 (19.12.1944), S. 1.

56 Informationen Pressekonferenz vom 20.12.1944, Nr. 36, in: BA Berlin, R 34/26.

57 Das OKW meldet, in: Der Angriff Nr. 309 (21.12.1944), S. 1. „Deutschland ist gefährlich“ - Feindstimmen zur Winterschlacht im Westen, in: Ebd. 10.000 Gefangene, 200 Panzer [...] Das Oberkommando der Wehrmacht gibt bekannt, in: VB 57. Jg./Nr. 342 (21.12.1944), S. 1. Illusionspolitik des Feindes bloßgestellt, in: Ebd. 
kontrovers diskutiert. Einen zwar gefärbten, aber dennoch umfangreichen Überblick bieten hierzu die Tagebücher des Bismarck-Vertrauten Moritz Busch. ${ }^{58}$

Im Ersten Weltkrieg war zwischen Militärs und Politikern verschiedener Couleur zunächst umstritten, ob die Kriegszieldiskussion ein militärisches oder ein politisches Thema sei. Im ersteren Fall wäre sie nicht erlaubt gewesen, im zweiten schon. Die militärischen, aber auch die Zivilbehörden vertraten zunächst eine äußerst restriktive Linie. ${ }^{59}$ Nach langer kontroverser Debatte wurden die Kriegsziele schließlich am 15.11.1916 zur öffentlichen Diskussion zugelassen. Das Zensurbuch von 1917 hielt fest: „Die sachliche Erörterung der Kriegsziele ist freigegeben. Verboten bleibt nur: 1. jede verhetzende Bekämpfung der Ansichten Andersdenkender oder ihrer Beweggründe; 2. jede Betrachtungsweise, die auf eine Beeinflussung der militärischen Kriegführung hinausläuft; 3. jede Störung des Verhältnisses zu unseren Verbündeten und den Neutralen." ${ }^{\circ 0}$ Obwohl diese Passagen nicht frei von dehnbaren Begriffen waren, war im Ersten Weltkrieg die Meinungsbildung ab der zweiten Kriegshälfte und bei den wichtigsten Themen möglich. Auch die Metakommunikation über den Sinn und Zweck, Schaden und Nutzen der freigegebenen Kriegszieldebatte selbst ist ein Indikator dafür, dass die Presse im Ersten Weltkrieg der Meinungsbildungsfunktion noch nachkommen konnte. Eine Probe, wie unterschiedlich die Berichterstattung der Blätter ausfallen konnte, lieferte die Erörterung der Friedensinitiative des Reichskanzlers vom 12.12.1916. Während die offiziöse NAZ - wohl auch von der Rücksicht auf die 3. OHL unter Hindenburg und Ludendorff diktiert - inhaltlich neutral mit „Kanzlerrede im Reichstage“ aufmachte und dem Angebot nur eine 15-zeilige Meldung widmete, ${ }^{61}$ beschäftigte sich das liberale „Berliner Tageblatt“ auf der ganzen 1. Seite mit dem „Friedensangebot des Deutschen Reiches und seiner Verbündeten“. Den Leitartikel schrieb Theodor Wolff. ${ }^{62}$ Ähnliche Unterschiede fanden sich auch in der regionalen Presse. ${ }^{63}$

Ganz anders sah das für den Zweiten Weltkrieg aus. Politik wurde im Führerhauptquartier gemacht. Wenn dennoch ganz enge Spielräume für die journalistischen Ausführungen blieben, dann deshalb, weil Goebbels dekretiert hatte: „Die Polyphonie der Zeitungen soll in jedem Fall erhalten bleiben." ${ }^{64}$ Ein anderer Grund für eine gewisse Vielgestaltigkeit der nationalsozialistischen Presse blieb auch im Krieg das sogenannte "polykratische Chaos“. Damit verlässt man jedoch schon die Sphäre journalistischer Autonomie und begibt sich in das Neben- und Durcheinander der propagandistischpolitischen Akteure hinter Hitler: Dietrich, Goebbels, Ribbentrop, Rosenberg u. a. ${ }^{65}$

58 Busch (Fn. 10), Bd. 2, S. 1-174. Vgl. die vorsichtige Position gegenüber „auswärtigen“, d. h. v. a. süddeutschen Blättern in der PC: Deutschlands Wünsche wegen Elsaß und Lothringen, in: PC 8. Jg./Nr. 35 (31.8.1870), S. 1.

59 Vgl. Bayer. Staatsministerium des Innern an Regierungen, Runderlass vom 22.8.1914 (TbNr. 2304), in: BaSTA, K3 Pr.-Reg., Nr. 2658, Nr. I.

60 Kommunikationsüberwachende Vorschriften des Jahres 1917 [Zensurbuch der Oberzensurstelle im Kriegspresseamt], in: Fischer, Heinz-Dietrich (Hg.): Pressekonzentration und Zensurpraxis im Ersten Weltkrieg. Texte und Quellen, Berlin 1973, S. 194-275, hier: S. $240 f$.

61 Das Friedensangebot, in: NAZ 56. Jg./Nr. 345 (13.12.1916), S. 1.

62 BT 45. Jg./Nr. 636 (12.12.1916), S. 1. Sösemann (Fn. 10), S. 461-463 (12.12.1916)

63 Ein deutsches Friedensangebot, in: BamVb 44. Jg./Nr. 343 (13.12.1916), S. 1; Die Aufnahme des Friedensangebots, in: Ebd. Nr. 344 (14.12.1916), S. 1. Vgl. Deutschlands Friedensangebot, in: BamTb 82. Jg./Nr. 343 (13.12.1916), S. 1; Graefe-Goldebec, A. v.: Kanzler wach auf! in: Ebd. 83. Jg./Nr. 4, S. 1 f.

64 Boelcke (Fn. 24), S. 36 (3.2.1940).

65 Stöber, Rudolf: Germany 1933-1945, as media case study, in: Johnston, Donald H. (Hg.): En- 


\section{Orientierungsfunktion}

Unmittelbarster Zweck journalistischer Orientierung sind Handreichungen für die Zukunft. Doch wie die Meinungsbildung könnte auch die Orientierungsfunktion als Summe aller anderen Medienfunktionen gelesen werden. Um eine Entgrenzung zu vermeiden, soll sie auf die Prognose des Zukünftigen eingeschränkt werden: Prognosen lassen Rückschlüsse auf anstehende Aktionen zu und verfehlte Prognosen haben gravierende Wirkungen auf die Stimmung der Öffentlichkeit; die Kommunikationskontrolle wird Prognostik daher nach Möglichkeit unterbinden.

Der Krieg von 1870/71 war zu kurz und zu erfolgreich, um journalistische Prognostik zu einem Konflikt werden zu lassen. Doch fehlte beispielsweise in der PC in den ersten Kriegsmonaten jeder Beitrag, der einen Blick in die Zukunft warf. Erst nach den ersten Siegen, unmittelbar vor der Entscheidungsschlacht von Sedan, findet sich ein Artikel, der auch in diese Kategorie passt. ${ }^{66}$ In der Folgezeit finden sich regelmäßig Artikel mit Ausblickscharakter. Aber ausgerechnet nach dem Krieg fand eine auf den ersten Blick nichtssagende Nachricht die Aufmerksamkeit der Behörde: „Frankreich. Unter den deutschen Occupationstruppen bemerkt man ein lebhaftes Hin- und Hermarschiren, welches als Vorbereitung zur Räumung zweier Departements betrachtet wird. “67 Vermutlich - die mehrbändige Akte gibt über die Beweggründe keine Auskunft - wurde der Artikel ausgeschnitten, weil er implizit die politische Absicht der Reichsregierung prognostizierte. Die nach dem preußischen Pressegesetz zuständige Behörde, wie in Bayern die Bezirksregierung - hier von Schleswig - sah zwar, liest man die Akte positivistisch, keinen Anlass einzuschreiten. Vielleicht aber ist die Redaktion mündlich ermahnt worden. Bei früherer Gelegenheit hatte Bismarck hingegen wegen prognostischer Artikel scharf reagiert. ${ }^{68}$ Der Vergleich der Vorgänge belegt zudem ganz en passant die Dominanz der politischen Kriterien in der Reichseinigungszeit.

Im Ersten Weltkrieg, im Zensurbuch von 1917, hieß es lapidar: „Prophezeiungen über den Erfolg schwebender Operationen müssen unterbleiben. “69 Ab 1917 waren insbesondere Prognosen zum U-Boot-Krieg unerwünscht. ${ }^{70}$ Und im Zweiten Weltkrieg, in Goebbels Ministerkonferenz, wurde der Presse wiederholt untersagt, Prognosen zu

cyclopedia of International Media and Communications, 4 Bde., Amsterdam/Boston/London/ New York et al. 2003, S. 221-235.

66 Deutschlands Wünsche wegen Elsaß und Lothringen, in: PC 8. Jg./Nr. 35 (31.8.1870), S. 1. In Bayern verhält es sich übrigens ähnlich. Nach einer statistischen Gegenüberstellung der deutschen und französischen Truppenstärken fragt z. B. das BamTb rhetorisch: „Wer kann bei dieser überlegenen Macht Deutschlands mit Grund noch Besorgnisse hegen?“, in: BamTb, Nr. 196 (20.7.1870), S. 1671. Wenn man will, könnte man dies als orientierende Mitteilung mit prognostischem Charakter lesen. In der Folgezeit finden sich aber nicht einmal Nachrichten, die solch allgemeine Prognosen abgeben. Das ändert sich erst, nachdem der Krieg praktisch entschieden ist. Vgl. BamTb, Nr. 230 (23.8.1870), S. 1835. BamTb, Nr. 240 (2.9.1870), S. 2011.

67 Ueternser Tageblatt, 24.9.1872, Nr. 11, S. 2. Vgl. Kreisblätter der Provinz Schleswig, GStArch I. HA, Rep. 77, tit. 972, Nr. 2, Bd. 1.

68 Die Berathungen der Bevollmächtigten der Norddeutschen Regierungen, in: PC 4. Jg./Nr. 52 (27.12.1866), S. 4. Die Eröffnung des Reichstags des Norddeutschen Bundes, in: PC 5. Jg./Nr. 5 (30.1.1867), S. 2. PrMinPräs an PrMI, Schreiben vom 30.1.1867, Tb II 1127, in: GStA I.HA, Rep 77, Tit 53, Nr. 64, Bd. 1, Bl. 102-105R. Vgl. PrMI an PrMinPräs, Schreiben vom 24.1.1867, Tb P.I. 129, in: Ebd., Bll. 99f. PrMinPräs an PrMI, Schreiben vom 2.2.1867, Tb II 1602, in: Ebd., Bl. 101. PrMinPräs an PrMI, Schreiben vom 2.2.1867, Tb II 1602, in: Ebd., Bl. 101.

69 Zensurbuch 1917, in: Fischer (Fn. 60), S. 255.

70 Ebd., S. 268. 
stellen: „Allgemein soll nochmals darauf hingewiesen werden, dass es Aufgabe der Presse lediglich sein darf, über Gewesenes zu berichten, und dass Voraussagen unter gar keinen Umständen zulässig sind. " ${ }^{71}$ Daraus könnte die Hypothese abgeleitet werden, dass Prognosen, wenn sie dennoch gestellt wurden, eine souveräne Hinwegsetzung der Journalisten über die Anweisungen anzeigten. Einige verfrühte Siegesmeldungen entpuppen sich jedoch als das genaue Gegenteil: als Ausdruck des Monopolanspruchs der politischen Führung. Unter der Hauptschlagzeile „Der Feldzug im Osten ist entschieden. Die große Stunde hat geschlagen“ meldete der VB am 10.10.1942 „Das militärische Ende des Bolschewismus“: „Heute vor einer Woche, in der Nacht vom 1. zum 2. Oktober, hat der Führer in einem Aufruf, dessen Wortlaut wir auf Seite 3 wiedergeben, die deutschen Soldaten der Ostfront aufgefordert ,zu dem letzten gewaltigen Hieb, der noch vor dem Einbruch des Winters diesen Gegner zerschmettern soll ${ }^{\circ}$ - aufgerufen zur , letzten großen Entscheidungsschlacht dieses Jahres'. Das war ein Befehl von einer Größe und Kühnheit wie kaum ein anderes Dokument der Kriegsgeschichte! Heute, eine einzige Woche später, meldet die Front Führer und Volk, daß der Krieg im wesentlichen vollzogen, daß die Entscheidung im Sinne dieses Befehls strategisch gefallen ist. Wenn jemals der Begriff des Blitzkrieges verwirklicht werden konnte - hier ist er verwirklicht worden! Sieben kurze Herbsttage haben genügt, um der ungeheuerlichsten Kriegsmaschine aller Zeiten den tödlichen Stoß zu versetzen, von dem sie sich niemals wieder erholen kann. " $" 72$

Die Berichterstattung enthüllte gleichermaßen den beschwörenden wie appellativen Charakter des Befehls. Der VB verdeutlichte den Kontext auf Seite 3, indem Hitler im Kreise der Soldaten gezeigt wurde; ein weiteres Foto dokumentierte die Reichspressekonferenz mit der voreiligen Verkündigung der "Zertrümmerung der Sowjetfront" durch Reichspressechef Otto Dietrich. Weitere zwei Fotos zeigten die vorrückenden deutschen Soldaten. Den größten Teil der dritten Seite nahm ein Foto Hitlers unter der Überschrift ein: „So rief der Führer zur Entscheidungsschlacht. Der historische Tagesbefehl an die Ostfront vom 2. Oktober" ${ }^{73}$

Am Folgetag hatte Hitler im Sportpalast zur Eröffnung des Winterhilfswerks erklärt, die Sowjetunion sei „bereits gebrochen“ und werde „sich nie mehr erheben“ “. ${ }^{74}$ In Goebbels Minister-Konferenz wurde hingegen auf deutliche Zurückhaltung gedrängt: Man solle „bei der Kommentierung der Führer-Rede [...] nicht über das hinausgehen, was der Führer wörtlich sagte. [...] Was das Kriegsende anbetrifft, so kommt es darauf an, herauszustreichen, daß es nicht so wichtig ist, wann der Krieg zu Ende ist, als vielmehr, wie er zu Ende geht. ${ }^{75}$ Der „Angriff“ machte das gleiche Thema deutlich zurückhaltender als der VB auf. Auch hier wurde am 10.10. auf Seite 3 ein Foto von Dietrich bei dessen Verkündigung „Der Feldzug im Osten ist entschieden!“ veröffentlicht, doch auf der Titelseite hieß es vorsichtiger: „Bolschewismus vor dem Ende“. Zudem war das eine untergeordnete Schlagzeile, die Hauptschlagzeilen lauteten: „England erkennt seine Ohn-

71 Boelcke (Fn. 24), S. 56 (24.5.1940).

72 Das militärische Ende des Bolschewismus, in: VB 54. Jg./Nr. 283 (10.10.1941), S. 1.

73 So rief der Führer zur Entscheidungsschlacht, in: VB 54. Jg./Nr. 283 (10.10.1941), S. 3. Silex verlegte die Konferenz in den November 1941. Silex, K.: SX., S. 221. Vgl. Abel, Karl-Dietrich: Presselenkung im NS-Staat, (Einzelveröffentlichungen der Historischen Kommission zu Berlin, Bd. 2), Berlin 1968, S. $19 f$.

74 Domarus, Max: Hitler. Reden und Proklamationen 1932-1945, Würzburg 1963, Bd. 2, S. 1758 1767, hier: S. 1763.

75 Boelcke (Fn. 24), S. 188 (4.10.1941). 
macht. Große Bestürzung in Washington“. Eine Karikatur „Des deutschen Schwertes Spitze“ zeigte den vom Schwert durchbohrten russischen Bären mit der Physiognomie Stalins. Die aus dem Brustkorb ragende Schwertspitze zielte auf einen zwergenhaften Churchill. $^{76}$

In der deutschen Öffentlichkeit folgte eine Phase großer Euphorie; Ende Oktober wich sie der Ernüchterung, da die Kampfhandlungen immer noch nicht beendet waren. Dabei überlieferte der SD zwar Kritik an Dietrich, nicht aber an Hitler - ein Beleg dafür, dass die „Meldungen aus dem Reich“ Informationen filterten. ${ }^{77}$ In der Jahresabschlusschronik des VB zum „Kriegsjahr 1941“ war folglich von der Entscheidung im Osten nicht mehr die Rede. Aus dem „historischen Tagesbefehl“ wurde unter dem Eintrag zum 2. Oktober 1941 der inhaltsleere Satz: „Tagesbefehl des Führers an die Soldaten der Ostfront." Auch die Niederlage vor Moskau verschwieg die Jahreschronik. ${ }^{78}$ Zum Jahresende brachte der „Angriff“ neben diversen Jahresrückblicken eine Betrachtung des Führers der Deutschen Arbeiterfront (DAF) Robert Ley zum Thema „General Zeit“, in der er den Nachweis zu führen suchte, dass die Zeit nicht für England, sondern für Deutschland arbeite. ${ }^{79}$ Ein indirektes Eingeständnis, dass erstens der Kampf in Russland keineswegs entschieden sei und zweitens die Vorbereitungen für den Ostfeldzug mangelhaft gewesen waren, steckte in dem Aufmacher vom Vortag: „Die ersten gesammelten Wollsachen rollen zur Ostfront. “80

Der Fehler der verfrühten Prognose wiederholte sich ein Jahr später in der Stalingrad-Berichterstattung, nachdem Hitler am 19.8.1942 den Angriff auf die Stadt an der Wolga befohlen hatte. Nach Dietrichs Tagesparole vom siegreichen Angriff auf Stalingrad räumte der VB in der zweiten Septemberhälfte 1942 der Stalingradberichterstattung breiten Raum ein. Aus einem Luftbild der brennenden Stadt hätte nur ein äußerst kritischer Zeitungsleser schließen können, dass die Bodentruppen Stalingrad längst noch nicht eingenommen hatten. ${ }^{81}$ Am gleichen Tag konnte man im „Angriff“ lesen: „Der schwere Kampf um Stalingrad neigt sich dem Ende zu. " 82 Wieder hatte Goebbels vergebens vor allzu optimistischer Berichterstattung über Stalingrad gewarnt. ${ }^{83}$

Hitlers Bürgerbräu-Rede, in der er behauptete, Stalingrad bereits zu besitzen, es sei kein zweites Verdun, bildete den Aufmacher des VB am folgenden Tag; die Bemerkungen zu Stalingrad aber wurden - vielleicht wegen der Erfahrungen aus dem Vorjahr - nicht gedruckt. Stattdessen hieß es in allgemeiner Form, dass von sowjetischer Seite „der Endsieg überhaupt nicht mehr gefährdet werden“ könne. ${ }^{84}$ Der „Angriff“ druckte die Rede im vollen Wortlaut inklusive all ihrer „prophetischen“ Passagen, u. a.: „Und alle unsere Gegner können überzeugt sein: Das Deutschland von einst hat um 3/4 12 die Waffen niedergelegt - ich höre grundsätzlich immer erst 5 Minuten nach 12 auf! [...] Sie werden sich noch der Reichstagssitzung erinnern, in der ich erklärte: Wenn das Judentum sich etwa einbildet, einen internationalen Weltkrieg zur Ausrottung der europäischen Rassen herbeiführen zu können, dann wird das Ergebnis nicht die Ausrottung der

76 Der Angriff Nr. 245 (10.10.1941), S. 1 und 3.

77 Boberach (Fn. 54), S. 2903 (23.10.1941); S. 2916 (27.10.1941); S. 2927 (30.10.1941).

78 Kriegsjahr 1941, in: VB 54. Jg./Nr. 365 (31.12.1941), S. 3.

79 Ley, Robert: General „Zeit“, in: Der Angriff Nr. 315 (31.12.1941), S. 1.

80 Der Angriff Nr. 314 (30.12.1941), S. 1.

$81 \mathrm{Vgl}$. Luftaufnahme vom brennenden Stalingrad, in: VB 55. Jg./Nr. 260 (17.9.1942), S. 3.

82 Die letzte Höhe vor Stalingrad, in: Der Angriff Nr. 226 (17.9.1942), S. 2.

83 Boelcke (Fn. 24), S. 188, S. 275-283 (24.8.-18.9.1942).

84 Der Führer sprach zum 9. November. Unser Kriegsplan wird eisern durchgeführt, in: VB 55. Jg./Nr. 313 (9.11.1942), S. 1f. 
europäischen Rassen, sondern die Ausrottung des Judentums in Europa sein. (Beifall) Man hat mich immer als Propheten ausgelacht. Von denen die damals lachten, lachen heute unzählige nicht mehr, und die, die jetzt noch lachen, werden es vielleicht in einiger Zeit auch nicht mehr tun. (Beifall) [...] Ich wollte zur Wolga kommen, und zwar an einer bestimmten Stelle, an einer bestimmten Stadt. Zufälligerweise trägt sie den Namen von Stalin selber. Aber denken Sie nicht, daß ich aus diesem Grunde dorthin marschiert bin [...] Dort schneidet man nämlich 30 Millionen Tonnen Verkehr ab [...] dort war ein gigantischer Umschlagplatz. Den wollte ich nehmen und - wissen Sie - wir sind bescheiden, wir haben ihn nämlich! Es sind nur noch ein paar ganz kleine Plätzchen da." ${ }^{85}$ Trotz der weitschweifigen Erklärungen zum Thema Stalingrad bestritt Hitler, wie schon bei der Belagerung Leningrads, ganz entschieden die Symbolträchtigkeit des Orts. ${ }^{86}$ In der Folgezeit wurde bis in die 2. Januarhälfte 1943 Stalingrad kaum erwähnt. Und selbst nach der endgültigen Kapitulation wurden die Tatsachen mehr verklärt und verschleiert als zugegeben. ${ }^{87}$

Die Prognose vom Herbst 1941 wurde ein letztes Mal in der Ardennen-Offensive bemüht. Diesmal, so hieß es, gäben die Alliierten falsche Prognosen über den weiteren Kriegsverlauf. ${ }^{88}$

\section{III.Zusammenfassung und weiterführende Überlegungen}

Was bleibt? Kriegsberichterstattung ist, da kann wohl jeder zustimmen, Teil des Krieges. Wenn man Krieg als Sonderfall der Politik und ergo Kriegsberichterstattung als Spezialfall politischer Kommunikation begreift - da mag nicht jeder zustimmen -, hat das nicht nur Konsequenzen für die Interpretation der journalistischen Autonomie, sondern eventuell auch für die Journalismusforschung.

Bei jeder der drei relevanten Funktionen - Informations-, Meinungsbildungs- und Orientierungsfunktion - zeigten sich zwischen den Kriegen signifikante Unterschiede.

Zur Informationsfunktion: Im Krieg von 1870/71 erlaubte die Engführung durch die Militärzensur den Journalisten in militärischen Belangen keine Bewegungsfreiheit. Für andere Fragen, die in den folgenden Kriegen Bedeutsamkeit erlangten, war der Krieg zu kurz - z. B. wurde Lebensmittelversorgung kein Thema. Im Wesentlichen schränkte die restriktive Informationspolitik des Militärs die journalistische Autonomie ein. Im Ersten Weltkrieg ließ sich hingegen an dem kriegsentscheidenden Thema des unbeschränkten U-Boot-Krieges zeigen, dass mit militärischer Zensur alleine der Informationsfluss nicht mehr zu steuern war. Wenn, wie im Falle Theodor Wolffs, ein Chefredakteur sein Blatt der Lenkung durch Kriegspresseamt, WTB und Berliner Pressekonferenz entziehen wollte, so konnte er zumindest durch Nichtberichten die intendierte Informationssteuerung unterlaufen. Im Zweiten Weltkrieg lagen die Medien eng am Gängelband. Verschweigen einerseits, Fehlinformationen andererseits und grundsätzliche Anweisungen, über dieses oder jenes in solcher oder anderer Aufmachung zu berichten, schränk-

85 Es geht um Sein oder Nichtsein. Die Rede des Führers zum 9. November in München, in: Der Angriff Nr. 271 (9.11.1942), S. 3-6, hier: S. 4. Vgl.: Domarus (Fn 74), S. 1935-1938.

$86 \mathrm{Zu}$ Leningrad vgl. die Rede vom 8.11.1941: Domarus (Fn 74), S. 1775.

87 Vgl. Der Angriff Nr. 29-31 (3.-5.2.1943), jeweils S. 1. Vgl. VB 56. Jg./Nr. 32-35, jeweils S. 1.

88 „Es ist gerade acht Tage her, seit der Reichspressechef in Wien den Satz sprach ,Im Jahre 1941 haben wir uns getäuscht, diesmal täuschen sich die anderen. 'Das ist ein Gedanke, den wir uns immer vor Augen halten müssen. “ Informationen Pressekonferenz vom 18.12.1944, Nr. 32, in: BA Berlin R 34/26. 
ten die Journalisten in einem kaum noch steigerbaren Maße ein. Die scheingenauesten Informationen in jedem Krieg boten die Statistiken. Für sich genommen, schienen große Zahlen für gegnerische Niederlagen und deutsche Siege zu sprechen. Doch waren sie ohne expliziten Zusammenhang nichts wert. Sofern, was die Regel war, gegnerische Verluste ohne eigene angegeben waren, fehlte der Maßstab. Im Zeitverlauf mussten aber selbst einordnungslose Zahlen auf das Publikum zumindest ermüdend wirken; zumeist regten sie darüber hinaus das Nachdenken an. Doch noch mehr als das Publikum zeigten sich die Medien als Statistiksüchtige. Wenn zur journalistischen Autonomie auch aufgeklärtes Selbstdenken gehört, so ließ es sich an den Medien jedenfalls nicht ablesen - mit der bemerkenswerten Ausnahme des BT.

Während bei der Informationsfunktion - abgesehen von der beschriebenen Ausnahme - keine signifikanten Unterschiede zwischen den ersten beiden Kriegen, wohl aber zwischen dem Ersten und Zweiten Weltkrieg - insbesondere wegen der positiven Pressepolitik im Nationalsozialismus - auszumachen waren, nahm die Autonomie in der Meinungsbildung von Krieg zu Krieg ab. Der deutsch-französische Krieg erlaubte der Presse noch divergierende Bewertungen. Im Ersten Weltkrieg wurde lange um das Wichtigste gerungen, die Freiheit zur politischen Meinungsbildung setzte sich schließlich zumindest in abstracto bei den großen Themen Frieden und Kriegsziele durch. Das zeigte sich in der überregionalen wie in der regionalen Presse. Im Zweiten Weltkrieg hingegen war der Spielraum der Medienakteure - selbst vordergründig betrachtet - äußerst marginal.

Bei der Orientierungsfunktion wiederum waren die Unterschiede zumindest vordergründig betrachtet gering. In allen drei Kriegen kamen Meldungen prognostischen Inhalts bisweilen vor, im ersten insbesondere, nachdem der Krieg entschieden war. Im Zweiten Weltkrieg zeigt sich jedoch auch hier das absolute Primat der politischen Führung. Die sensationellsten Meldungen der Kriegsberichterstattung - der Russlandfeldzug ist entschieden (1941) und Stalingrad ist erobert (1942) - gingen auf Äußerungen Hitlers zurück. Die journalistische Autonomie beschränkte sich nur noch auf die sensationellere oder weniger sensationelle Aufmachung.

Alle drei Funktionen zusammengenommen zeigen, dass die journalistische Autonomie immer dort an ihre Grenzen stieß, wo sie mit den übergeordneten politischen Interessen kollidierte. Auch dies ist nochmals Beleg für die These, dass über Kriegsberichterstattung, Propaganda und politische Kommunikation nicht geredet werden kann, wenn zuvor nicht das Verhältnis von Krieg und Politik bestimmt ist. Für die journalistische Autonomie in der Kriegsberichterstattung war dabei nicht die oft bemühte Zensur das Gefährlichste, sondern die sogenannte positive Pressepolitik, d. h. Medienlenkung durch ereignisbezogene Anweisungen. Stufte die Zensur der Kriege von 1870/71 und 1914-1918 die autonome Kriegsberichterstattung zu einem Faktor zweiter Größe zurück, so konnte der Journalismus unter den Bedingungen der nationalsozialistischen positiven Presselenkung nur noch als Faktor dritter Größe gelten. Die Politik bedrohte die Autonomie gerade dadurch, dass sie sich journalistische Routinen zunutze machte und die Funktion der Presse in propagandistischem Interesse instrumentalisierte.

Angesichts der Randbedingungen der drei Kriege lassen sich darüber hinaus jedoch keine allgemeingültigen Aussagen formulieren. Je stärker eigene nationale Interessen die Kriegsberichterstattung beeinflussen und je weniger frei das Mediensystem insgesamt ist, desto geringer fällt die journalistische Berichterstattungsautonomie aus. Sie mag ohne Einschränkungen in Demokratien gelten, die nicht an dem Krieg beteiligt sind, über den gerade berichtet wird. Sie mag mit gewissen Einschränkungen für die Berichterstattung in kriegsbeteiligten Demokratien gelten; doch schon das Beispiel der angloamerikani- 
schen Demokratien im 20. und 21. Jahrhundert lehrt, dass Pressefreiheit - und das heißt auch journalistische Gestaltungshoheit - in Kriegszeiten nur eingeschränkt existiert. In den drei deutschen Kriegswirklichkeiten zwischen 1870/71, 1914/18 und 1939/45 nahm sie tendenziell ab, bis sie am Schluss nicht mehr vorhanden war.

Damit aber sei der heutigen Journalismusforschung - zumindest dem systemtheoretischen Mainstream - die provokante Frage gestellt, ob deren arbiträre Versuche, das journalistische System über Codes zu definieren, nicht leer laufen müssen? Wenn z. B. die Beachtung der Regel öffentlich/nicht öffentlich den Journalismus definierte, stünde dann Kriegsberichterstattung nicht eigentlich außerhalb des journalistischen Systems? Oder wären die Propaganda- und Zensurstellen Teile eines Subsystems? Das Problem gilt mutatis mutandis auch für die anderen Codes wie Information/Nicht-Information, publizieren/nicht publizieren, aktuell/nicht aktuell. Nun könnte man, mit Gödels Unvollständigkeitssatz oder Poppers Unvollständigkeitstheorem argumentierend, ${ }^{89}$ die Codes zu Axiomen des journalistischen/publizistischen Systems erklären. Weitere Diskussionen über voraussetzungslose, nicht beweisbare, konkurrierende Apriori besäßen dann allerdings eher philosophischen als wissenschaftlichen Charakter. Erachtete man hingegen die jeweiligen Codes als systemisches Kriterium im Sinne Carl Schmitts, stellte sich das Gödel-Poppersche Problem vor dem Hintergrund der System-Umwelt-Beziehung erneut. Aus diesem Paradox gibt es keinen Ausweg; Paradoxa deuten aber auf grundsätzliche Argumentationsfehler. Was dabei wie ein Fundamentalangriff auf die Systemtheorie aussieht, entpuppt sich bei näherer Betrachtung als eine Ehrenrettung; denn ein Generalvorwurf lautet, die Systemtheorie ließe sich nicht falsifizieren. Das mag zwar im Großen und Ganzen richtig sein, bei konkreten, empirisch beobachtbaren System-Umweltbeziehungen lassen sich systemtheoretische Teilaussagen aber durchaus falsifizieren. So ermöglicht auch der diachrone Vergleich keinen Ausweg aus dem Dilemma, macht aber zumindest darauf aufmerksam.

89 Popper, Karl R.: Wissenschaftliche Reduktion und die essentielle Unvollständigkeit der Wissenschaft, in: Ders.: Alles Leben ist Problemlösen. Über Erkenntnis, Geschichte und Politik, 6. Aufl., München/Zürich 1995, S. 47-92. 